\title{
Remarkable response to fluorouracil, leucovorin, oxaliplatin, and irinotecan therapy in urothelial cancer of the renal pelvis: a case report
}

Takuya Tsujino, Kiyoshi Takahara*, Tomohisa Matsunaga, Yuki Yoshikawa, Tomoaki Takai, Taizo Uchimoto, Kenkichi Saito, Naoki Tanda, Hajime Hirano, Hayahito Nomi, Naokazu Ibuki, Teruo Inamoto and Haruhito Azuma

\begin{abstract}
Background: No standard chemotherapy regimen for advanced urothelial cancer has been established, except for cisplatin-based regimens. We report the case of a patient with double primary cancer, urothelial carcinoma of the upper urinary tract and colorectal cancer, who underwent oxaliplatin-based chemotherapies.

Case presentation: A 56-year-old Japanese man presented to our hospital with the diagnosis of a left renal pelvic tumor and rectal cancer. Several examinations including ureteroscopic biopsy and computed tomography-guided biopsy were performed; however, the diagnosis of renal pelvic cancer could not be made. Because the rectal cancer had been growing during the course of examination, he underwent five cycles of neoadjuvant chemotherapy with fluorouracil, leucovorin, oxaliplatin, and irinotecan. The volumes of both the rectal cancer and renal pelvic tumor drastically decreased. He then underwent pelvic evisceration with colostomy and ureterocutaneostomy. The histological diagnosis of the renal pelvic tumor was urothelial carcinoma. He is free of disease at 12 months after the treatment.

Conclusions: To the best of our knowledge, this is the first report describing a remarkable response to fluorouracil, leucovorin, oxaliplatin, and irinotecan therapy for renal pelvic cancer. We suggest fluorouracil, leucovorin, oxaliplatin, and irinotecan is an effective therapy for patients with advanced urothelial cancer.
\end{abstract}

Keywords: Urothelial carcinoma, Upper tract urothelial carcinoma, FOLFOXIRI, Oxaliplatin, Fluorouracil, Irinotecan, Case report

\section{Background}

Most cases of urothelial carcinoma (UC) develop in the urinary bladder, while $\mathrm{UC}$ of the upper urinary tract (UTUC) is uncommon, accounting for only 5 to $10 \%$ of all renal tumors. Radical nephroureterectomy with bladder cuff excision remains the standard treatment for patients with UTUC with large, multifocal or high-grade tumors. The effect of perioperative chemotherapy for UTUC remains unclear. We report the remarkable anti-tumor effect of oxaliplatin-based chemotherapies for UTUC.

\footnotetext{
* Correspondence: uro061@osaka-med.ac.jp; uro037@osaka-med.ac.jp Department of Urology, Osaka Medical College, 2-7 Daigaku-cho, Takatsuki, Osaka 569-8686, Japan

\section{Case presentation}

A 56-year-old Japanese man with a diagnosis of a left renal pelvic tumor and rectal cancer was referred to our hospital. He had been smoking cigarettes for 36 years. He had been healthy all his life and his family history was unremarkable. His chief complaints were bloody stools and proctodynia for several months. On admission, his vital signs were normal. An abdominal examination revealed distension; a rectal examination showed bloody stool and narrowing of his anus. The findings on other examinations were completely unremarkable. Laboratory examination revealed anemia and an inflammatory response (hemoglobin level of $100 \mathrm{~g} / \mathrm{L}$, white blood cell count of $12.5 \times 10^{9} / \mathrm{L}$, neutrophil count 
of $10.8 \times 10^{9} / \mathrm{L}$, and lymphocyte count of $1.3 \times 10^{9} / \mathrm{L}$ ); the other findings were normal. His level of carcinoembryonic antigen (CEA) was normal. Contrast-enhanced abdominal computed tomography $(\mathrm{CT})$ showed a huge mass in his rectum and a left renal pelvic tumor. Histological examination of a sample obtained during colonoscopy revealed adenocarcinoma of the rectum. Several examinations including ureteroscopic and CT-guided biopsies were performed; however, the diagnosis of renal pelvic cancer could not be made. Because the rectal cancer was growing during our evaluations of the patient, we started to administer two cycles of neoadjuvant chemotherapy with fluorouracil, leucovorin, oxaliplatin, and irinotecan (FOLFOXIRI). The volumes of the rectal cancer and renal pelvic tumor decreased drastically at the end of the two cycles of chemotherapy (Fig. 1). He received a total of five cycles of FOLFOXIRI. The renal pelvic tumor reduced by $50 \%$ as measured across the maximum diameter (from $64 \mathrm{~mm}$ to $32 \mathrm{~mm}$ ). During the administration of chemotherapy, laboratory examination revealed neutropenia (white blood cell count of $1.7 \times 10^{9} / \mathrm{L}$ with $0.46 \times 10^{9} / \mathrm{L}$ neutrophils). Febrile neutropenia was observed at the end of the first round of chemotherapy; however, no other severe adverse events were observed after that. Subsequently, laparoscopic total nephroureterectomy on the left side was performed following pelvic evisceration with colostomy and ureterocutaneostomy on the right side. On histological examination, the rectal cancer and renal pelvic tumor were diagnosed as adenocarcinoma (pT2N0M0) and UC (pT3N0M0) with high grade, respectively, as presented in Fig. 2. There has been no disease progression at 12 months after treatment.

\section{Discussion}

UC responds very well to cisplatin-based chemotherapy, represented by gemcitabine-cisplatin (GC) therapy; therefore, this treatment is widely administered to patients with UC. However, effective chemotherapy regimens, other than cisplatin-based chemotherapies, for patients with UC have not been established, although many clinical trials are ongoing. Oxaliplatin is more effective than cisplatin in vitro, and has shown efficacy in preclinical studies using many tumor cell lines [1]. In addition, Siew et al. demonstrated the efficacy of oxaliplatin, an immunogenic cell death inducer, on the induction of stress ligands and promotion of natural killer cellmediated cytotoxicity in human ovarian cancer [2]. The

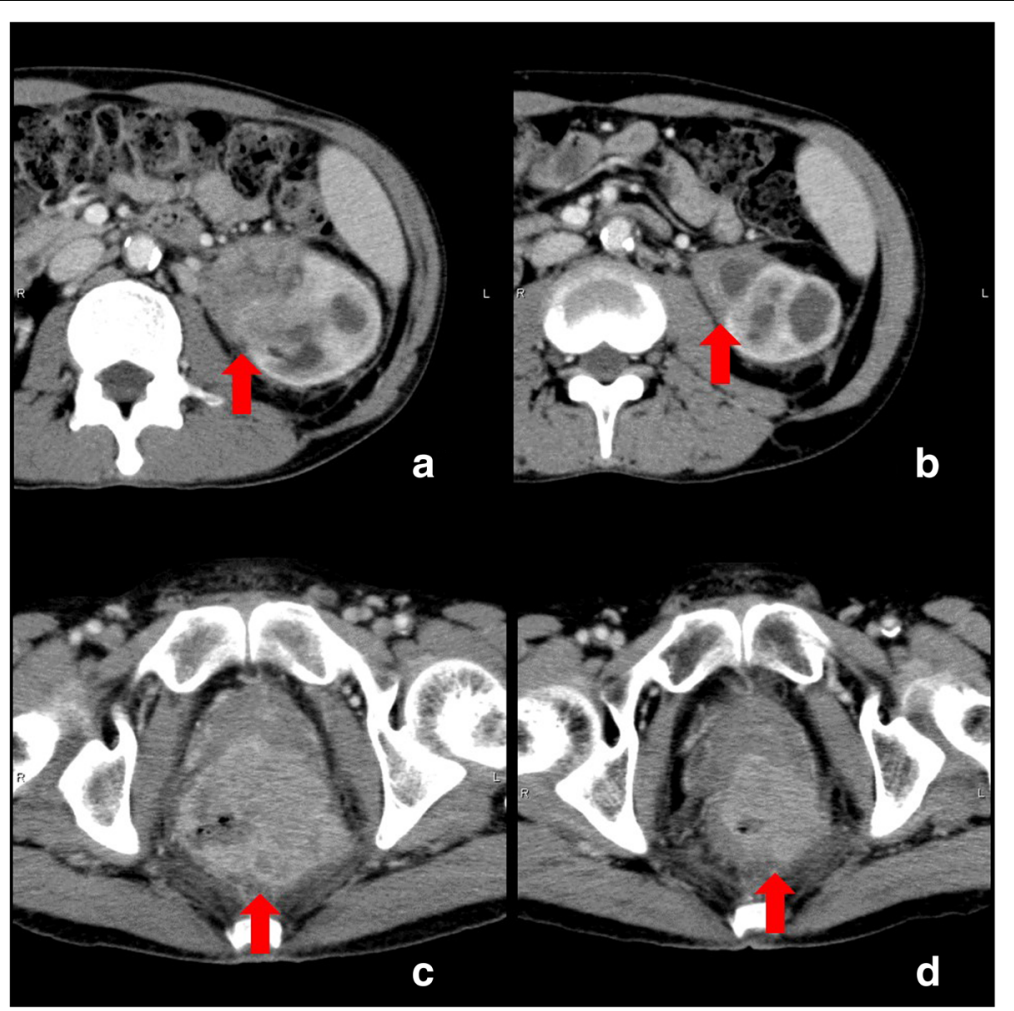

Fig. 1 a, b Abdominal computed tomography scan demonstrating the volume of renal pelvic tumor before treatment (a), and the decrease in volume after two cycles of fluorouracil, leucovorin, oxaliplatin, and irinotecan (b). c, $\mathbf{d}$ The rectal cancer before treatment (c), and decrease in tumor volume after two rounds of fluorouracil, leucovorin, oxaliplatin, and irinotecan (d). Red arrows indicate the sites of the tumor 


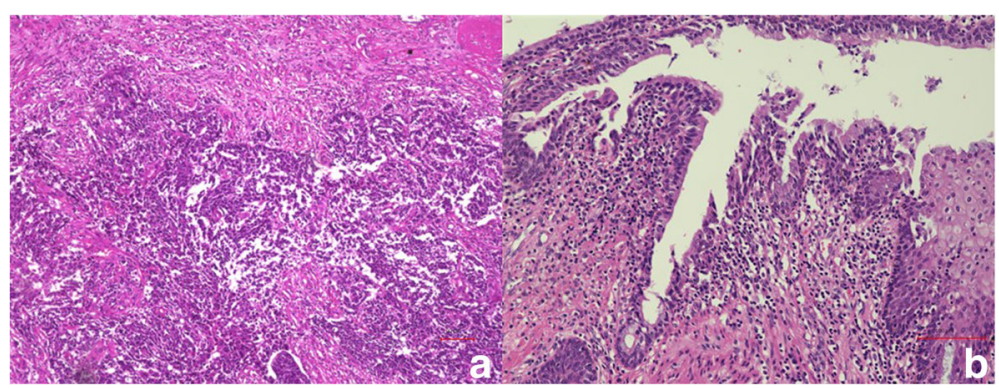

Fig. 2 a The renal pelvis tumor was histologically diagnosed as high-grade urothelial carcinoma (hematoxylin and eosin, $\times 100$ ). b The rectal cancer revealed adenocarcinoma (hematoxylin and eosin, $\times 200$ )

efficacy of an oxaliplatin alone chemotherapy for advanced or metastatic UC was minimal in phase II studies. Those studies showed response rates of not more than several percent $[3,4]$. The efficacy of 5-fluorouracil (5-FU) for advanced UC remains unclear, but a review of published studies in 1987 described response rates of approximately $15 \%$ using the unmodulated single agent 5-FU [5]. In addition, a phase II trial of continuous 5-FU infusion in 2009 showed a median progression-free survival of 1.9 months, a median overall survival of 6.5 months, and a response rate of $20 \%$ [6].

In 2008, a phase II study was performed to determine the efficacy of irinotecan monotherapy for advanced transitional cell carcinoma of the urothelium; the median progression-free survival was 2.1 months and the median overall survival was 5.4 months [7]. However, these monotherapy regimens do not usually give good responses for cancers, including colorectal cancer. The response rates of irinotecan, oxaliplatin, and 5-FU were only approximately $18 \%, 20 \%$, and $21 \%$, respectively. On the other hand, combination therapies using a combination of fluorouracil, leucovorin, and irinotecan (FOLFIRI), a combination of fluorouracil, leucovorin, and oxaliplatin (FOLFOX), and FOLFOXIRI had stronger activities in colorectal carcinoma [8-11]. There are some reports on the use of combination therapies for patients with UC. The case report about the use of FOLFIRI for UC by Lu et al. showed that UTUC was well controlled by the chemotherapy regimen, and, that it was effective for metastatic colorectal cancer [12]. There are few reports of FOLFOX therapy for UC, with only a phase II trial by Lorenzo et al. [13]. They used FOLFOX in 18 patients who had previously been treated for UC, and reported only low-grade toxicity and a $19 \%$ overall response rate [13]. The case report of FOLFOX for UC by Seo et al. showed a complete response in a patient who developed lung metastasis and an additional primary colon cancer after radical nephrectomy for the UC [14]. In our patient with UC, chemotherapy with FOLFOXIRI resulted in an extremely good response.

\section{Conclusions}

To the best of our knowledge, this is the first report describing a remarkable response to FOLFOXIRI therapy in a renal pelvic cancer and UC. Therefore, we suggest FOLFOXIRI therapy is a novel and effective therapy for advanced UC patients.

\section{Acknowledgements}

Not applicable.

Funding

No funds were necessary for this study.

Availability of data and materials

As the study was a case report, materials and data are the medical file of the patient. The pictures can be obtained from our institution.

\section{Authors' contributions}

All the authors contributed to the workup of the patient including clinical evaluation, investigation, and treatment planning. The manuscript has been reviewed and approved by all the authors.

\section{Competing interests}

The authors declare that they have no competing interests.

\section{Consent for publication}

Written informed consent was obtained from the patient for publication of this case report and any accompanying images. A copy of the written consent is available for review by the Editor-in-Chief of this journal.

Ethics approval and consent to participate

Not applicable.

\section{Publisher's Note}

Springer Nature remains neutral with regard to jurisdictional claims in published maps and institutional affiliations.

Received: 20 December 2016 Accepted: 9 March 2017

Published online: 10 April 2017

\section{References}

1. Rixe O, Ortuzar W, Alvarez M, Parker R, Reed E, Paull K, et al. Oxaliplatin, tetraplatin, cisplatin, and carboplatin: spectrum of activity in drug-resistant cell lines and in the cell lines of the National Cancer Institute's Anticancer Drug Screen panel. Biochem Pharmacol. 1996;52:1855-65.

2. Siew YY, Neo SY, Yew HC, Lim SW, Ng YC, Lew SM, et al. Oxaliplatin regulates expression of stress ligands in ovarian cancer cells and modulates their susceptibility to natural killer cell-mediated cytotoxicity. Int Immunol. 2015;27(12):621-32. dxv041.

3. Moore MJ, Winquist E, Vokes EE, Hirte H, Hoving K, Stadler WM. Phase II study of oxaliplatin in patients with inoperable, locally advanced or 
metastatic transitional cell carcinoma of the urothelial tract (TCC) who have received prior chemotherapy. In Proc Am Soc Clin Oncol. 2003;22:408.

4. Winquist E, Vokes E, Moore MJ, Schumm LP, Hoving K, Stadler WM. A Phase II study of oxaliplatin in urothelial cancer. Urol Oncol. 2005:23:150-4.

5. Yagoda A. Chemotherapy of urothelial tract tumors. Cancer. 1987;60:574-85.

6. Highley MS, Griffiths GO, Uscinska BM, Huddart RA, Barber BP, Parmar MB,

Bladder Cancer Clinical Studies Group, et al. A phase II trial of continuous 5fluorouracil in recurrent or metastatic transitional cell carcinoma of the urinary tract. Clin Oncol. 2009;21:394-400.

7. Beer TM, Goldman B, Nichols CR, Petrylak DP, Agarwal M, Ryan CW, Crawford ED. Southwest Oncology Group phase II study of irinotecan in patients with advanced transitional cell carcinoma of the urothelium that progressed after platinum-based chemotherapy. Clin Genitourin Cancer. 2008;6:36-9.

8. Saltz LB, Cox JV, Blanke C, Rosen LS, Fehrenbacher L, Moore MJ, Elfring GL, Irinotecan Study Group. Irinotecan plus fluorouracil and leucovorin for metastatic colorectal cancer. N Engl J Med. 2000;343:905-14.

9. Diaz-Rubio E, Sastre J, Zaniboni A, Labianca R, Cortes-Funes H, De Braud F, Homerin M. Oxaliplatin as single agent in previously untreated colorectal carcinoma patients: a phase II multicentric study. Ann Oncol. 1998;9:105-8.

10. de Gramont AD, Figer A, Seymour M, Homerin M, Hmissi A, Cassidy J, Papamichael D. Leucovorin and fluorouracil with or without oxaliplatin as first-line treatment in advanced colorectal cancer. J Clin Oncol. 2000;18:2938-47.

11. Falcone A, Ricci S, Brunetti I, Pfanner E, Allegrini G, Barbara C, Cortesi E. Phase III trial of infusional fluorouracil, leucovorin, oxaliplatin, and irinotecan (FOLFOXIRI) compared with infusional fluorouracil, leucovorin, and irinotecan (FOLFIRI) as first-line treatment for metastatic colorectal cancer: the Gruppo Oncologico Nord Ovest. J Clin Oncol. 2007;25:1670-6.

12. Lu YM, Chien TM, Lin CH, Chai CY, Huang CN. Epidermal growth factor receptor inhibitor with fluorouracil, leucovorin, and irinotecan as an alternative treatment for advanced upper tract urothelial carcinoma: a case report. J Med Case Reports. 2016;10:1.

13. Di Lorenzo G, Autorino R, Giordan A, Giuliano M, D'Armiento M, Bianco AR, De Placido S. FOLFOX-4 in pre-treated patients with advanced transitional cell carcinoma of the bladder. Jpn J Clin Oncol. 2004;34:747-50.

14. Seo R, Kim SH, Kim H, Kim CK, Park SK, Koh ES, Hong DS. Complete response to FOLFOX4 therapy in a patient with advanced urothelial cancer: a case report. J Hematol Oncol. 2010:31:1.

\section{Submit your next manuscript to BioMed Central and we will help you at every step:}

- We accept pre-submission inquiries

- Our selector tool helps you to find the most relevant journal

- We provide round the clock customer support

- Convenient online submission

- Thorough peer review

- Inclusion in PubMed and all major indexing services

- Maximum visibility for your research

Submit your manuscript at www.biomedcentral.com/submit

C Biomed Central 